RESEARCH PAPER RP879

Part of Journal of Research of the National Bureau of Standards, Volume 16, April 1936

\title{
GLOSS INVESTIGATIONS USING REFLECTED IMAGES OF A TARGET PATTERN
}

\author{
By Richard S. Hunter
}

\section{ABSTRACT}

A target pattern of concentric rings varying from fine lines to broad bands has been placed in the open face of a desk lamp. This luminous target is useful in the study of the gloss characteristics of the more glossy surfaces. The lines and bands of various sizes in the pattern provide means for studying surfaces of a wide range of "distinctness-of-reflected-image" gloss. Records may be made of which lines and bands are visible by reflection from different surfaces. Such records serve as permanent gloss values for the different surfaces studied. The dark areas of the target immediately adjacent to the luminous areas provide ideal conditions for the identification of surface "bloom." The best gloss differentiations are made when the lamp is used in a darkened room, so that the luminous pattern is the only source of light illuminating the surfaces inspected. Photographic records of gloss and unusual gloss effects are discussed.

I. Introduction

II. Apparatus and method

III. Representative results

IV. Identification of differences in bloom, objective gloss, and texture... 363

V. Value of gloss photographs

VI. General technique 366

\section{INTRODUCTION}

In a recent circular ${ }^{1}$ of the scientific section of the National Paint, Varnish, and Lacquer Association, the author described an instrument to measure "distinctness-of-reflected-image" gloss and to study other gloss effects. In this gloss comparator two identical, illuminated targets consisting of concentric rings varying from fine lines to wide bands were used. The narrowest lines which could be seen after reflection from any given surface were used as the criterion of the distinctness-of-image gloss of that surface. A measuring device which gave variable amounts of diffusion to one of the targets was used to arrange these distinctness-of-image comparisons on a continuous scale.

Shortly after this gloss comparator was introduced, it was realized that perhaps the luminous target itself, without the accompanying instrument, might be useful in gloss-control work and studies of gloss differences. Accordingly, an enlargement of the gloss-comparator target was printed on a photographic plate and mounted in the face

1 Scientific Section Circular 493 of the NPVLA, Washington, D. C. (October 1935). In this circular are described the gloss comparator and also the different appearance effects identified with glossiness. A. bibliography on gloss-measuring methods is included. 
of a desk lamp, as illustrated in figure 1. This lamp has proved so useful in studies of gloss that it is thought worth-while to describe its use in detail.

\section{APPARATUS AND METHOD}

In figure 2, a 90-degree quadrant of the target is reproduced to full scale. Each of the lines and bands in the target is designated with a letter by which it may be identified. The advantages which the device offers when used for studies of gloss and gloss differences are: (1) it affords reproducible conditions for the study of glossy materials; (2) the lines and bands of varying size in the target provide means for studying surfaces over a wide range of distinctness-of-image gloss; (3) permanent records of this type of gloss may be obtained by identification of the rings and bands which are visible after reflection from any given surface; and (4) the dark areas in the target may be used for determining the differences in bloom between glossy surfaces.

The design of the circular target was reached through a trial-anderror development during the building of the earlier instrument. ${ }^{2}$ Rectangular and straight-line targets were tried first, but it was discovered that the images reflected by many surfaces varied as the surfaces were rotated in their own plane. The circular target, of course, gives the imaging powers from source lines in all directions. The widths of the lines and bands have been graded to give fairly even steps in distinctness-of-image reflection. To illustrate the differentiations thus made possible, three pairs of typical target images have been photographed with the combination of camera and lamp shown in figure 1. These photographs are reproduced in figure 3.

The lamp makes possible the finest distinctions in gloss when it is used in an otherwise darkened room. However, since the ordinary glossy appearance of any surface varies with the surrounding field of view and with other illuminations, it is, in many cases, advisable to make gloss comparisons with outside light present in order to realize more closely the actual conditions of gloss observation. It is best to obtain flat panels of the materials to be studied as curved surfaces reflect distorted images, which are more difficult to evaluate. A mild example of this distortion is seen in figure 3 , where the panel a-1 possesses a slight curvature.

For detecting gloss differences, the two panels to be compared are placed side by side and viewed so that part of the image of the face of the lamp is seen reflected from each surface. The distance of the surfaces from the lamp and the angle of view are always important and should be noted where permanent records are to be kept. If the test panels are moved toward the lamp, finer lines in the target may be distinguished. Also, a shift of the surfaces toward the lamp will make their lightness and color characteristics more pronounced. When the surfaces are moved away from the lamp, the finer lines in the pattern disappear, and color and lightness become more difficult to recognize. As the angle of view approaches grazing incidence, images reflected in the surfaces become more distinct. Thus many of the very flat, mat surfaces will reflect distinguishable images at near-grazing incidence.

When the lamp is used as a source for obtaining distinctness-ofimage gloss ratings of individual surfaces, instead of simply for com-

\footnotetext{
2 See footnote 1.
} 


$$
\text { C- }
$$


paring two surfaces, these surfaces must, in turn, all be placed in the same position relative to the lamp and should be viewed from the same position. The exterior-lighting conditions must also be constant and the following factors specified in presenting the results:

1. Distance from lamp to test surface.

2. Distance from observer to test surface.

3. Direction of view (unless otherwise specified, approximately $45^{\circ}$ ).

4. Amount of light present from sources outside the lamp. When these factors are recorded, reproducible conditions for the identification of the lines of the target are established, and observations made

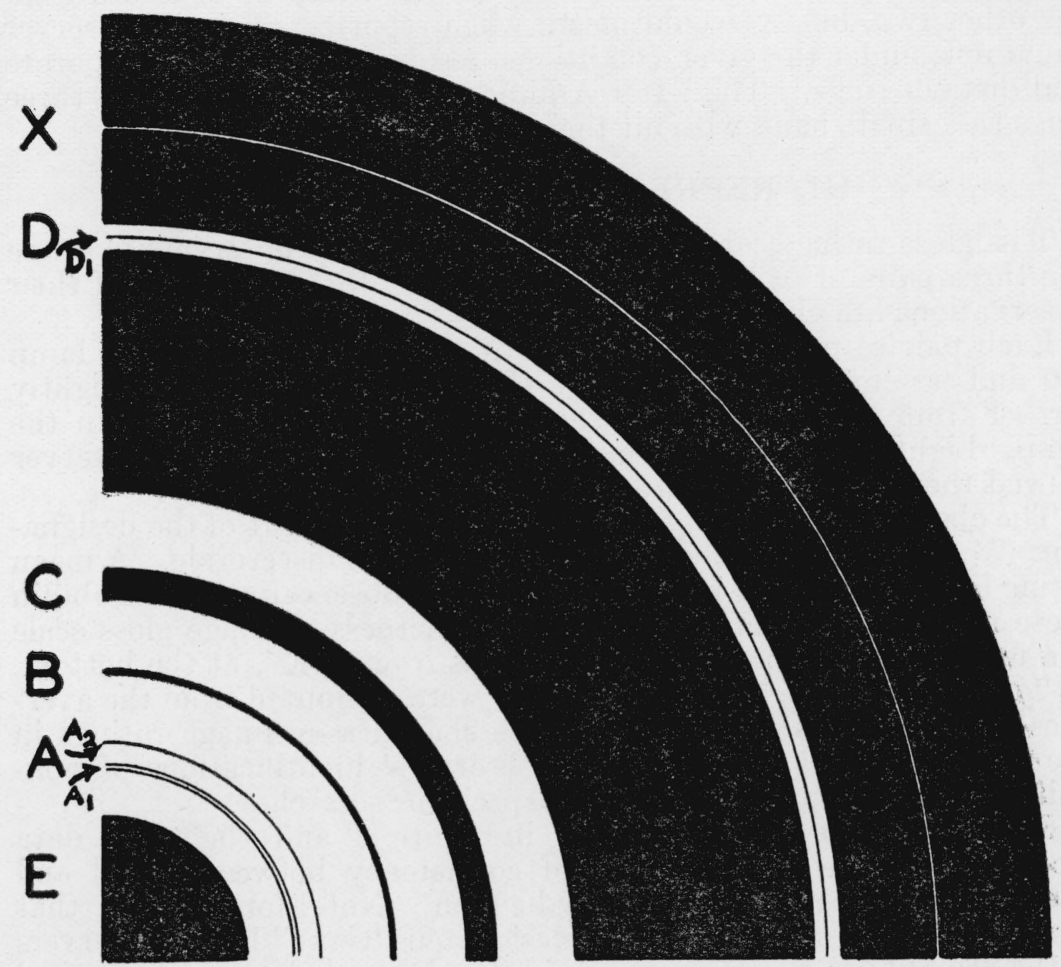

FIGURE 2.-One-quarter circle of the target pattern reproduced full scale, with letters to identify the different rings and lines.

at separate times may be used as records of the gloss of the surfaces tested.

The observer who wishes to describe the appearance of the target. image reflected in some particular glossy surface, names and describes the appearance of the least-distinct ring. That is, he records the letter which identifies this ring and perhaps a modifying letter to indicate whether this ring is sharp, fairly plain, or indistinct; also whether it appears as a continuous ring, or shows only irregularly from. certain parts of the surface. In order of descending distinctness-ofimage, the letters identifying the rings in the target are $A_{1}, A_{2}, A, B$, $\mathrm{C}, \mathrm{D}$, and $\mathrm{E}$. In addition, there are two auxiliary rings $\left(\mathrm{D}_{1}\right.$ and $\left.\mathrm{X}\right)$, which are occasionally used in describing the appearance of surfaces. 
The following modifying letters are suggested for the above designations: g, good definition; $f$, fair definition; and $p$, poor definition. This system gives a distinctness-of-image gloss scale of 7 letter ratings and 21 steps, 3 steps for each rating. Three letters, which serve to indicate the appearance of a surface aside from its distinctness-ofimage gloss, are suggested: n, nonuniform; w, wavy; and s, specks and scratches. Other characteristics of surfaces, such as bloom, particular types of texture, and unusual appearance may also be designated.

In the innermost band of rings, the letter $\mathrm{A}_{1}$ represents resolution by the eye of the two rings close together just outside the center of the target. $\mathrm{A}_{2}$ represents resolution of the third ring as separate from the other two, but $A_{2}$ would mean, when reported, that the observer could not, under the given conditions, resolve the $A_{1}$ pair as separate and distinct rings. The letter $\mathrm{A}$ indicates identification of the three rings as a single band without their separate resolution.

\section{REPRESENTATIVE RESULTS}

This gloss-rating scale was used by a number of observers to grade the three pairs of panels illustrated in figure 3 . The results of their observations are given in table 1.

Each pair of panels was observed at two distances from the lamp $(30$ and $90 \mathrm{~cm})$; in each instance the observer's eyes were slightly farther from the test surfaces than the test surfaces were from the lamp. Light was incident upon the specimens at $45^{\circ}$, and the observer viewed the images reflected at $45^{\circ}$; the room was dark.

The observer's rating, $g, f$, or $p$, is placed to the right of the designation $\left(A_{1}, A_{2}, \ldots.\right)$ of the ring which was least discernible. A mean rating is assigned at the bottom of each set of observations. To obtain these means, it is assumed that the distinctness-of-image gloss scale is a uniform, continuous scale of 21 steps from " $p \mathrm{E}$ ", at the bottom, to " $g \mathrm{~A}_{1}$ ", at the top. Tenths of a step were computed from the averages of the six observers. Below the sharpness-of-image ratings in the table are " $x$ 's" to indicate the observers' identifications of nonuniformities, surface waviness, and specks or scratches.

From the photographic record in figure 3 and the gloss data recorded in table 1, the degree of consistency between visual and photographic records of gloss may be seen. Note, for instance, that panels a-l and b-r, figure 3, were designated "wavy" by all observers in the visual test; also these panels in the photograph show the typical distortion due to waviness. The distinctness of the images reproduced in the figure do not exactly correspond to the $30-\mathrm{cm}$ ratings given in table 1, because of slight changes in reproduction introduced in the various printing processes.

When it is considered that the gloss-inspection lamp and the method of rating gloss were both new to these observers, the agreement among them seems quite satisfactory. The lamp is shown to be capable of establishing 21 steps on a distinctness-of-image scale. The range of this scale may be adjusted to give the best gloss differentiations of any particular type of glossy material. This adjustment is usually accomplished by varying the distances of lamp to test surface and test surface to observer. Glossy surfaces which reflect sharp images should be compared at greater distances from the lamp, while better differentiations are made on the less-glossy surfaces by moving them closer to the lamp. The average, observed, 
distinctness-of-image, gloss-step differences, expressed in gloss steps between the $l$ and $r$ panels of each pair at the two distances, tabulated in table 1, are as follows:

\begin{tabular}{|c|r|r|}
\hline & \multicolumn{2}{|c|}{ Distances } \\
\cline { 2 - 3 } Samples & $30 \mathrm{~cm}$ & $90 \mathrm{~cm}$ \\
\hline "a" pair.... & & \\
\hline "b" pair & 0.5 & 2.3 \\
& .3 & 1.0 \\
& 4.8 & 3.0 \\
\hline
\end{tabular}

Thus it can be seen that the glossy " $\mathrm{a}$ " pair of panels showed an average difference of only one-half step at $30 \mathrm{~cm}$, while at $90 \mathrm{~cm}$ this difference was more than two steps. The converse is true for the "c" pair of low-gloss panels; thus, viewed at $30 \mathrm{~cm}$, the average difference was 4.8 steps, but at $90 \mathrm{~cm}$ the difference recognized was only 3.0 steps.

The preparation recently of a specification for the gloss of automobile enamels furnishes an example of the practical use of this flexible gloss scale. Representative test samples of such glossy enamels, some weathered and some fresh, were used for trial observations to determine under what conditions the best gloss differentiations could be made. It was found that at a lamp-to-surface distance of 1 meter, a lamp-to-observer distance of 1.5 meters, and an angle of view equal to $30^{\circ}$, practically the complete gloss scale could be used. The test room was dark, the lamp was placed on a bench, the test panels were placed on the floor, and the observers viewed the images of the lamp target reflected in the various surfaces while standing. Tentatively, the distinctness-of-image gloss requirements were chosen as follows:

Fresh surfaces: B fair, or higher.

After 168 hours of accelerated weathering: $\mathrm{C}$ good, or higher.

In comparing the superior and inferior enamels, it was found that the better-grade materials retained their gloss more effectively through accelerated weathering.

\section{IDENTIFICATION OF DIFFERENCES IN BLOOM, OBJECTIVE GLOSS, AND TEXTURE}

Bloom, objective gloss, and surface texture were three of the types of gloss identified in the earlier publication. ${ }^{3}$ It is not possible to arrange, by means of the lamp, these factors along a scale such as used in the case of distinctness-of-image gloss. However, the lamp and target have proved useful in identifying differences in these three types of surface appearance and in furnishing qualitative, rather than quantitative, data on them.

When using the gloss-inspection lamp for the identification of bloom, one inspects the image of the black spot in the center of the target and the other dark areas in the target. Bloom is indicated as a haze in these dark areas of the image. The two glossy olive-green enamel panels compared in figure 3, "a" pair, for distinctness-of-image 
TABLE 1.-Tabulation of distinctness-of-image gloss ratings by six observers of the three pairs of panels compared in figure $S$

[Panels were observed at two distances and surface characteristics noted]

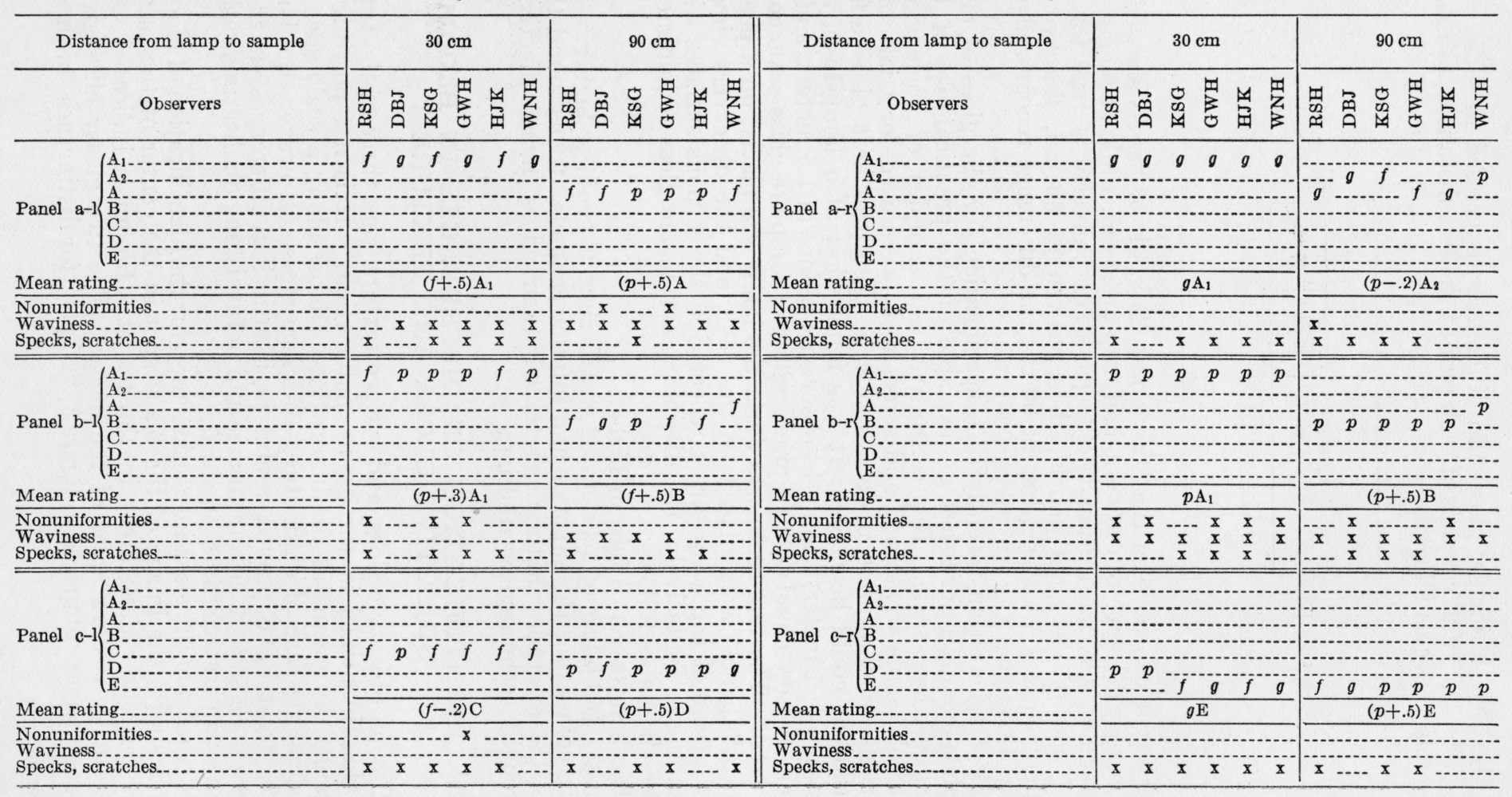




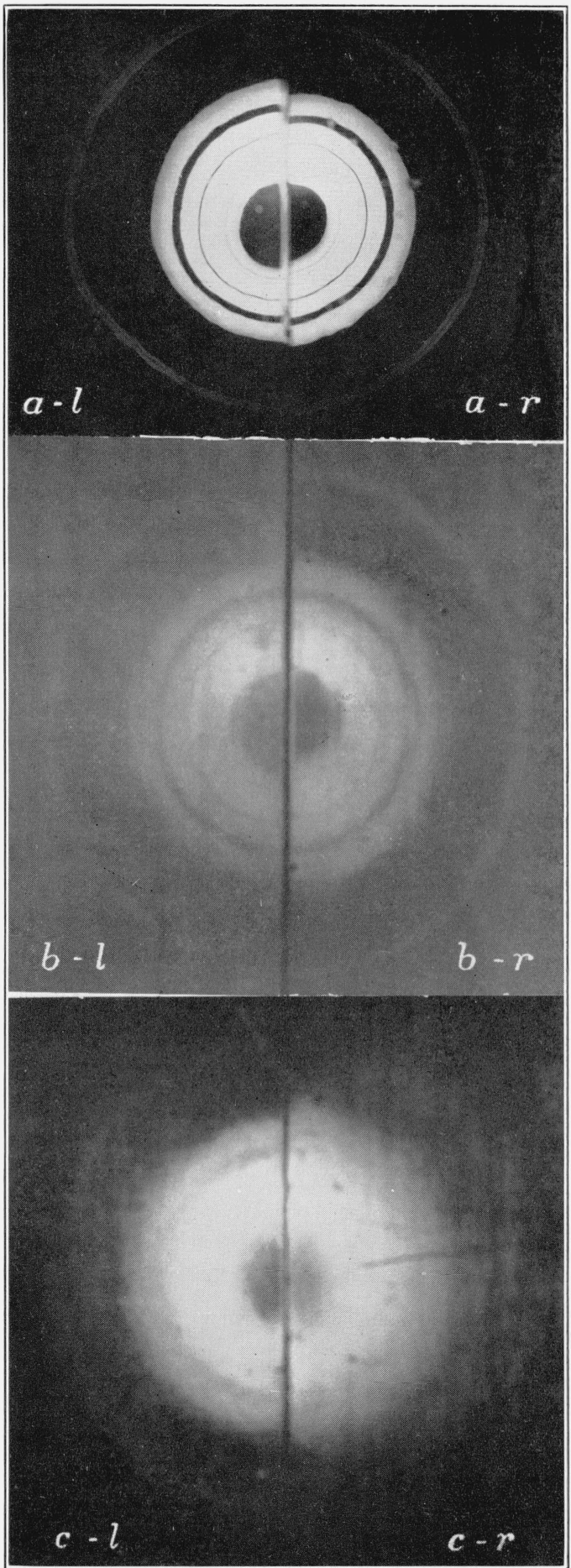

Figure 3.-Comparison of three pairs of panels using the gloss-inspection lamp. Panels, a, olive-green enamels; b, white, resinous paints; and c, rubbed furniture finishes. Distances of camera to surface and lamp to surface both about $30 \mathrm{~cm}$; room dark. 


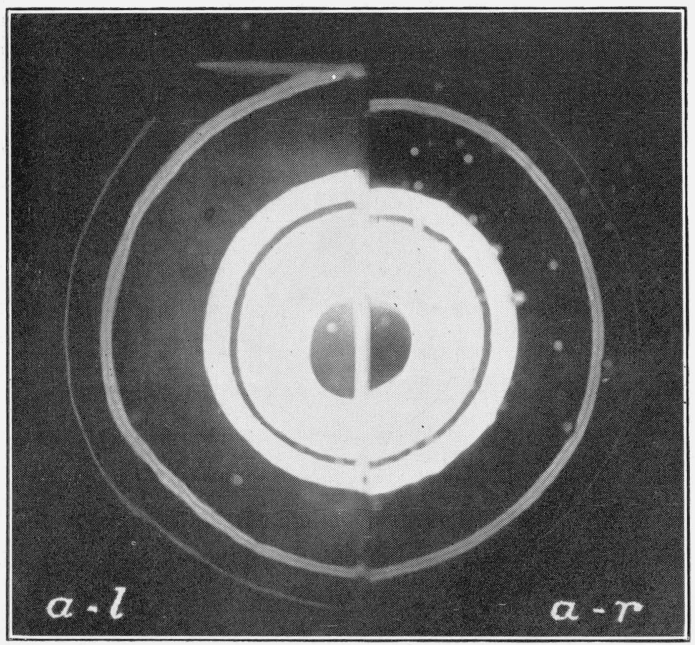

FIGURE 4.-Comparison of "a" pair of panels shown in figure 3 printed from same negative as in figure 3, but with low density to show effect of bloom on panel a-l. 
gloss, showed also a pronounced difference in bloom. The effect is quite appreciable in the left panel of figure 4, which is another print from the "a" pair negative of figure 3 , but less dense. In the " $b$ " pair of white surfaces, figure $3, \mathrm{~b}-\mathrm{l}$ reflects the sharper images, and also shows more bloom than b-r. Bloom is shown only by surfaces which reflect images.

Objective gloss is more popularly thought of as "shininess." With the gloss lamp and target, it is recognized by the brilliance of reflected images but is not readily measurable. There are a number of other gloss meters, however, which give records of specular reflectance, such as, for instance, the instrument ${ }^{4}$ designed by the author in the spring of 1934. References to methods for measuring objective gloss, as well as other gloss factors, are given in the previous circular. ${ }^{5}$

With regard to comparisons of surface texture and the evaluation of irregularities, this lamp is useful as a source of nearly unidirectional light. Nonuniformities, waviness, and specks or scratches were evaluated qualitatively by the observers who compared the six surfaces and obtained the results shown in table 1. Surface irregularities, blemishes, and texture show up distinctly in illumination from a single direction but are less visible in illumination which is well diffused. Texture is most distinct at the angle of specular reflection, and photographs at this angle with the camera focused on the surface tested, give, in our experience, the best records of texture. All the surfaces photographed in figure 3 show irregularities, but they are out of focus because the camera was focused on the image of the target.

\section{VALUE OF GLOSS PHOTOGRAPHS}

It can be appreciated that photographs such as are shown in figures 3 and 4 are better and more complete records of glossiness and serve better for comparing gloss than do values obtained on any gloss scale. This is because the glossiness of a surface arises from a combination of several factors and these combined factors are seldom expressible as a variation in one dimension. Because of the advantages of the photographic method, the earlier gloss comparator ${ }^{6}$ was designed to take a camera directly into the instrument. The camera is always placed in the same position in the instrument; no careful adjusting and focusing are necessary, and the exposures are timed by a prepared table. With the gloss-inspection lamp, on the other hand, it is necessary to set the camera up carefully in the desired position to photograph the effect sought. In spite of the greater care which is necessary, this ability to use the camera in any position is sometimes an advantage because it gives greater latitude for the study of gloss effects.

A 5 by 7 plate camera was used to obtain the photographs shown in figures 3 and 4 . This was set up as shown in figure 1 , where the camera lens and the lamp target are both about $30 \mathrm{~cm}$ from the gloss surfaces. The lens of the camera was stopped-down to about $3 \mathrm{~mm}$ so that as much detail as possible would be obtained from the out-offocus test surfaces.

4 The Glossmeter, Scientific Section Circular 456 of the NPVLA, Washington, D. C. (April 1934).
s See footnote 1.

o See footnote 1.
6 See footnote 1 . 


\section{GENERAL TECHNIQUE}

As already implied, the observer who wishes to analyze gloss effects must recognize that two distinctly different modes of appearance, as well as several appearance effects, are always possible. One mode results when the observer fixates the glossy surface itself; the other when he fixates the image of some object reflected in that surface. Surface texture, formation, and structure are appreciated when the eye is focused upon the surfaces being studied. On the other hand, form of reflected images, gloss contrasts, and the distribution of light reflected by surfaces are usually appreciated when the observer fixates the images of objects reflected in the glossy surfaces. For this reason, one who wishes to photograph surface features and texture focuses the camera upon the glossy surface, while one who wishes to photograph image reflections, bloom, or contrasts, as in figure 3 , focuses the camera upon the image of the target reflected in the test specimens. In this latter case, pimples, irregularities, and grain in the surfaces can be seen, but they are out of focus.

In conclusion, it may be repeated that the gloss-inspection lamp is a convenient source of light for the general study of gloss-appearance effects. The wide variety of ways in which it can be used makes it possible to identify many effects that are not appreciated when using other apparatus. Thus, as described in section II, the distances of the test surface from the lamp and observer and the surrounding lighting can be varied to obtain the conditions which give best discriminations of distinctness-of-image gloss for the particular type of surfaces being studied. Furthermore, unusual gloss effects can often be seen which do not fall directly into one of the classes identified in the previous publication. ${ }^{7}$ By using the gloss-inspection lamp to study these effects, and trying different methods of illuminating and viewing the samples, satisfactory conditions for the identification of many effects may be found.

For example, an unusual effect which appeared in comparing the "c" pair of furniture finishes photographed in figure 3 may be described. When these two surfaces were compared from a direction considerably to one side of the direction of image reflection shown, panel c-r, which shows distinctly the lower distinctness-of-image gloss, appeared to have a cleaner and glossier finish than panel c-l. The effect on c-l, which appeared to be similar to bloom or reflection haze, detracted considerably from its glossiness, and yet it was only identified under rather exceptional conditions of illumination.

Directional-distribution measurements of reflection as recommended by McNicholas ${ }^{8}$ may eventually lead to a more definite understanding of the conditions under which some of these appearance effects are likely to occur, and such measurements are now in progress; but for the present, empirical methods which are discovered by trial, using devices such as this lamp, offer the best means of identifying and recording the presence of unusual gloss types. Because there is so much latitude in establishing external conditions for the use of this lamp, it is believed that it will prove valuable in examining many types of gloss effects.

Washington, February 10, 1936.

7 See footnote 1.

- Absolute methods in reflectometry, BS J. Research 1, 29 (1928) RP3. 